\title{
Silver and gold nanoparticles as an integral part of nanooncology: current state of the problem
}

\author{
M.A. Osadchuk ${ }^{a^{*}}$, N.V. Kireeva ${ }^{a}$, N.P. Korzhenkov ${ }^{a}$ and M.V. Trushin ${ }^{b}$
}

${ }^{a}$ The Federal State Autonomous Education Institution of Higher Training The First Sechenov Moscow State Medical University under Ministry of Health of the Russian Federation (Sechenovskiy University), 119991, st. Trubetskaya, 8, building 2, Moscow, Russia ${ }^{b}$ Kazan Federal University, Kazan, Russia

\author{
C H R O N I C L E \\ Article history: \\ Received June 1, 2020 \\ Received in revised form \\ July 26, 2020 \\ Accepted September 25, 2020 \\ Available online \\ September 25, 2020 \\ Keywords: \\ Synthesis and properties of gold \\ and silver nanoparticles \\ Diagnosis and treatment of \\ cancer \\ Genotoxicity \\ Antigenotoxicity \\ Nanooncology
}

\begin{abstract}
A B S T R A C T
Gold and silver nanoparticles are easily synthesized and the most effective nanostructures in clinical practice. Their optical properties, ease of synthesis, colloidal stability and the ability to form any surface for easier conjugation with biological particles, the ability to individual multiple use, make them especially important in oncology. Gold and silver nanoparticles are capable of providing targeted drug delivery, which depends on the photothermal, photodynamic, and antiangiogenic properties of the metal. Due to their exceptional properties, these nanoparticles are considered as a potential tool for the diagnosis of various types of cancer and drug delivery. The non-toxic and non-immunogenic nature of gold and silver nanoparticles, as well as their high permeability and retention effect, provide additional advantages, allowing drugs to easily penetrate and accumulate at tumor sites. The effectiveness of precious metal nanoparticles as radiosensitizers, dose enhancers and contrast agents is gaining increasing recognition. An important problem associated with the use of biogenic metal nanoparticles is the study of their genotoxicity, since they play an important role in the initiation and progression of abnormalities, including genetic ones.
\end{abstract}

\section{Cancer is the main problem of the 21 st century}

At this stage of civilized world development, cancer is the main threat to human health with high mortality rates ${ }^{1}$. Globally, there has been an alarming incidence growth of all cancer types from 12.7 million new cases in 2008 to 22.2 million by $2030^{2,3}$. In this regard, development of a strategy for complete cure of cancer is a major public health objective. Currently, the literature shows a great deal of interest in tumor diagnosis and treatment based on nanoparticles (NPs) ${ }^{4-6}$. Clinically oriented nanotechnology studies give reason to speak about the formation of a new effective nanoscience and nano-oncology model ${ }^{7}$. In this regard, the rapidly developing nanotechnology opens up great opportunities for improving diagnostics and antitumor therapy quality ${ }^{3,7}$.

\section{Nanotechnology, nanomedicine, nano-oncology and general characteristics of nanoparticles}

Nanotechnology is an interdisciplinary science based on a combination of theoretical justification, analysis and synthesis, as well as methods for producing NPs with a given atomic structure, about 1- 
100 nanometers (nm) in size, for use in medicine, biology, physics, chemistry and mechanical engineering $^{8,9}$. By 2020 the global nanoindustry is expected to reach $\$ 75.8$ billion $^{10}$, and by 2026 the sale of NPs will amount to $\$ 50$ billion ${ }^{11}$.

Nanomedicine is a translational science, whose purpose is to obtain new therapeutic and diagnostic tools using modern nanotechnology ${ }^{12}$. It is aimed at developing methods for delivering drugs to certain human body tissues, increasing diagnosis, imaging and therapy effectiveness. The use of nanotechnology in designing new drug delivery systems is one of the most rapidly developing nanomedical research areas. NPs widespread use for both diagnosis and treatment of various localization cancer, contributed to the formation of a new medical sphere - teragnostics ${ }^{13}$. Thus, NPs development for cancer diagnosis and treatment has given rise to a new field of research - oncological nanomedicine. Nano-oncology`s goal is to detect, target and treat cancer cells without any side effects.

\section{Methods of gold and silver nanoparticles synthesis in oncology}

MNPs synthesis is a new nanoscience field due to their unique physical, electrical, chemical and optical properties ${ }^{9}$. In practice, two approaches to NPs synthesis are usually used: a top-down approach and a bottom-up one. They are shown in Fig. 1.

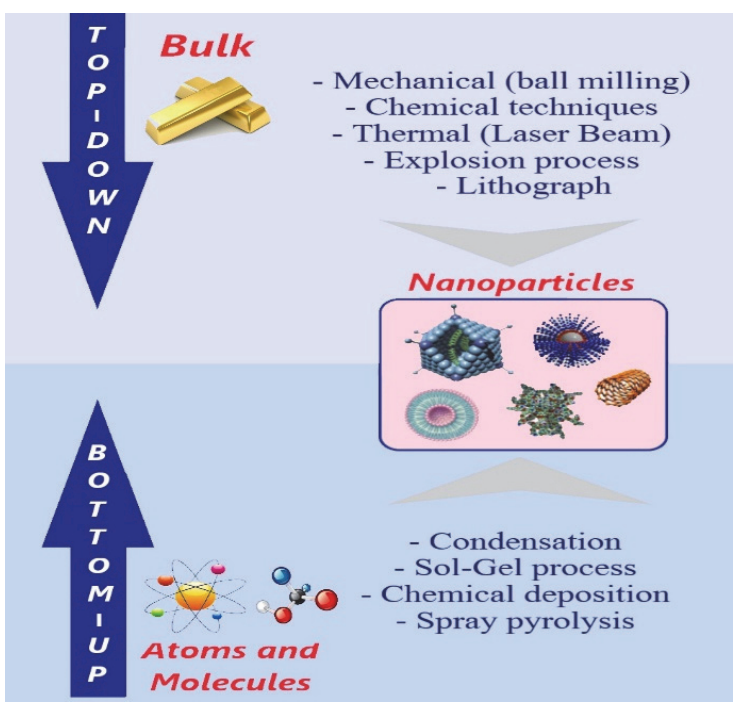

Fig. 1. ${ }^{14}$ Top-down and bottom-up approaches using various physical, chemical and biological methods for NPs synthesis

In the top-down approach to synthesis NPs are formed by cutting loose material by various mechanical means to obtain nanoscale structures. In the bottom-up approach NPs are synthesized at the molecular level using various chemical or biological agents ${ }^{10}{ }^{15}$. The modern method for MNPs synthesis is based on cations recovery with a possibility to adjust the size and shape ${ }^{16}$. In chemical synthesis methods, gold $(\mathrm{Au})$ ions in salts are recovered using citrate ${ }^{17,18}$, ascorbate ${ }^{19}$, borohydride ${ }^{20}$ or amines ${ }^{21}$. In these methods, the use of stabilizers is necessary to prevent gold nanoparticles (AuNPs) aggregation. Among various stabilizing agents, citrate ${ }^{22}$ and alkanethiols ${ }^{23}$ are considered universal agents. Since NPs differ not only in size and shape, but also in optical and electrical parameters, obtaining particles with uniform characteristics is crucial. Therefore, to control NPs size and shape, a certain temperature ${ }^{21}$, microwaves ${ }^{23}$, or UV irradiation ${ }^{24}$ are used. The biological method for nanostructures synthesis is free from a number of unsatisfactory conditions typical of physical and chemical methods for producing NPs (high temperature and energy requirements, hazardous wastes and toxic chemicals formation $)^{25-27}$. In this connection, nanobiotechnological studies belong to "gold biotechnologies" and suggest NPs synthesis by using only biological sources ${ }^{26}$. 
AuNPs are easily synthesized and most potentially effective nanostructures ${ }^{28}$. AuNPs optical properties, ease of synthesis, colloidal stability, and the ability to form NPs surface for easier coupling with biological particles make them especially relevant for biomedical applications. AuNPs and silver nanoparticles (AgNPs) alloys can be synthesized in both directions: Ag-coated by $\mathrm{Au}$ and / or Aucoated by Ag colloidal particles. This can be achieved by applying one metal salt to an already formed surface of another metal NPs. In addition, it is possible to control and produce various compositions of these bimetallic NPs by controlling the amount of salt deposited on preformed colloids ${ }^{29}$. It has been shown that they are substrates for surface-enhanced spectroscopy of combined light scattering and, therefore, can be used to detect drugs, proteins and biomolecules in body fluids, and to detect early cancer biomarkers ${ }^{30}$. In another study, Au-Ag NPs were used to detect different DNA strands in a single reaction system. This molecular nano-diagnostic approach can be implemented as an operational diagnostic test for cancer ${ }^{31}$. Au and Ag alloys limit AgNPs cytotoxicity and demonstrate a more increased plasmonic and photothermal activity than pure AuNPs, which makes it possible to consider them quite effective anticancer agents ${ }^{32}$.

\section{Nanoparticle classification and properties}

NPs can be classified into metal, polymer, carbon, magnetic, liposomes, dendrimers, and quantum depending on their type ${ }^{33}$. NPs can be clusters of ions, atoms, or molecules ${ }^{34}$. At the same time, their size is not strictly limited. So, NPs used for drug delivery, loaded with drugs, have a diameter of more than $100 \mathrm{~nm}^{35}$. Thus, the ideal NPs size for cancer treatment is $70-200 \mathrm{~nm}$, since fenestration in the tumor tissue endothelium is about $200-780 \mathrm{~nm}^{36}$. At the same time, NPs less than $100 \mathrm{~nm}$ have excellent ability to target the tumor, being small enough to penetrate from porous vascular endothelial fenestrations that surround the tumor area ${ }^{29}$. Unlike traditional anticancer drugs, with a number of significant side effects, NPs provide a strictly localized approach that prevents unwanted effects. NPs have specific physicochemical properties that are different from their volume, micro, or macro-sized counterparts, which are determined by UV-visible spectroscopy, Scanning Electron Microscope (SEM) and dynamic light scattering (DLS). It is of interest to consider these NPs formation mechanism in an aquatic environment through various stages of reduction, nucleation, and growth.

Characteristic by UV-visible spectroscopy. MNPs have optical properties that depend on NPs size, composition, and morphology. These optical properties are the result of surface plasmon resonance (SPR). When a metal is irradiated by an electromagnetic wave in the UV or visible spectrum the cumulative effect of free electrons oscillating coherently generates plasmons ${ }^{37}$. Gold and silver absorb light in the visible range, so the solution takes on colors that depend on the particle shape. Triangular particles are identified by red, pentagonal - by green, and spherical - by blue. The metal size, shape, composition and the NPs concentration affect the wavelength at which the absorption peak appears ${ }^{38}$. Based on this, it can be concluded that NPs consisting of a certain metal, size and shape will correspond to one or another absorption peak in the UV spectrum. Dynamic light scattering (DLS) is the primary analytical method used to characterize NPs in order to identify their size and distribution in the nanometer range. It is known that in a colloidal solution particle move in Brownian motion. When such a solution is irradiated with light, these suspended particles scatter the light at different angles, which is recorded by DLS. In this case DLS measures the autocorrelation coefficient or the time-dependent intensity function ${ }^{39,40}$.

Characteristic by microscopy. Being in the nanometer range, NPs can be monitored using SEM, transmission electron microscopy (TEM), scanning tunneling microscopy (STM), and atomic force microscopy (AFM). SEM is a NPs surface visualization method that uses an electron beam to obtain results in the form of signals corresponding to atomic composition features and other topographic data. SEM can achieve a resolution equal to or less than $1 \mathrm{~nm}^{41-42}$. TEM gives the same results as SEM, but uses a very thin portion of the sample through which the incident electron ray passes ${ }^{41}$. TEM is 
preferable to SEM, since it provides better spatial resolution and possibility of additional analytical measurements ${ }^{43}$.

\section{Gold and silver nanoparticles in diagnosis and treatment of cancer}

NPs with their unique physicochemical characteristics have pronounced antitumor properties. The NPs unique antitumor action is caused either by internal features associated with their direct antioxidant effect, or by the use of external stimuli connected with local hyperthermia in response to the use of infrared rays or magnetic fields. When exposed to external stimuli, a reactive oxygen species production occurs which causes cancer cell death. NPs can also interact with the tumor environment (blood vessels or stroma), and block developing the tumor itself ${ }^{44}$. $\mathrm{Au}$ and $\mathrm{Ag}$ possess pronounced antitumor properties. Of particular importance is the study of AuNPs and AgNPs potential for drug delivery, hyperthermia, bioimaging, photothermal therapy, cancer therapy itself and biosensor analysis. Owing to their small size, NPs can easily interact with biomolecules both on the surface and inside the cells, providing better signals and targeted specificity in cancer diagnosis and treatment. Basic chemicals with rapid metabolism used to treat tumors are toxic to the body and have significant side effects with drug resistance development. At the same time, NPs biological synthesis provides a fairly high safety and effectiveness of cancer treatment. NPs obtained using precious metals can have different properties and therefore, display one or another toxicity mechanism in relation to certain cancer cells. AuNPs and AgNPs can also be used in combination with drugs, which significantly improves therapy results ${ }^{45}$. AgNPs enter mammalian cells as aggregates, mainly through endocytosis and can also penetrate the blood-brain barrier. When they enter the cell in the endocytic vesicle by means of intracellular transport, they are distributed in the cytoplasm and nucleus ${ }^{46}$. Due to difference in their physicochemical properties, AgNPs can affect different cell types through various cellular processes. In this respect, NPs can be toxic not only to cancer cells, but to some extent, to normal cell structures ${ }^{47}$.

It was found that AgNPs possess chemical stability, high electrical conductivity, thermal conductivity, catalytic activity, and enhanced raman scattering. Mechanistic transcriptome analysis of cancer-associated fibroblasts (CAFs) showed that Ag-based nanomaterials cause expression changes in genes associated with cancer invasion and tumor metastasis ${ }^{48}$. MNPs can impact the tumor stroma carcinogenic activity by affecting stromal fibroblast genes expression and secretory profiles and thereby change the internal cross-connections with malignant cells. The AgNPs antitumor effect is associated with reactive oxygen species (ROS) production, lactate dehydrogenase (LDH) release, decreased mitochondrial function, cell cycle deregulation, induction of apoptotic genes such as Bax (Bcl-2-associated X protein), micronuclei formation, chromosomal aberrations and DNA damage ${ }^{49,50}$. This potential of metallic nanomaterials should be used in multimodal approaches to treatment and achieve better therapeutic results. AgNPs absorption by macrophages is especially evident in inflammation, often associated with the tumor process. These activated macrophages release ROS, tumor necrosis factor alfa (TNFa), inflammatory cytokines and interleukins (IL-6) ${ }^{51}$. AgNPs small sizes were more toxic and more effective in reactive ROS production ${ }^{52,53}$. In addition to these cellular mechanisms, AgNPs also demonstrated antianginal ${ }^{54}$ and antiproliferative ${ }^{55}$ properties. AgNPs are antiangiogenic because they inhibit phosphorylation of signaling pathway Akt by PI3K Akt by (protein kinase B) PI3K (phosphatidylinositol 3-kinase), which is incomplete in nature, blocking angiogenesis, depriving the cell of oxygen and thereby destroying the tumor cell ${ }^{54}$. The antitumor activity is due to AuNPs intrinsic properties, which enable them to selectively interact with heparin-binding glycoproteins and block their activity. The AuNPs ability to specifically bind to vascular permeability factor (VPF), vascular endothelial growth factor (VEGF), major fibroblast growth factor, endothelial cell mitogens and angiogenesis mediators inhibits endothelial fibroblast cell proliferation and angiogenesis ${ }^{56}$. AuNPs block phosphorylation of downstream molecules, such as Akt, ERK $1 / 2$ in the PI3K/Akt signaling pathway, ${ }^{67}$. Once inside the cells, AuNPs target tumor suppressor genes and oncogenes to induce efficient caspase- 9 expression $^{58}$. Core-targeted AuNPs contribute to blocking the 
cell cycle and inhibiting cytokines, which then initiate apoptosis ${ }^{59}$. In many cases AuNPs are used as a delivery system [60] or in conjugation with a therapeutic molecule ${ }^{61}$. AuNPs exhibit independent antitumor activity, due to their ability to provide targeted drug delivery, depending on the metal photothermal, photodynamic and anti-angiogenic properties ${ }^{5,28}$. In photothermal therapy AuNPs are used as a probe because of their strong absorption due to surface plasmon resonance( SPR) in the near infrared region, which leads to heating effects, followed by irradiation with a non-ionizing energy source, such as a laser. When laser irradiation is applied to AuNPs the SPR band is converted to heat, which causes hyperthermia ultimately leading to cell necrosis ${ }^{62}$. Photodynamic therapy is based on using a photosensitizer, such as 5-aminolevulinic acid, which is stimulated by irradiation and reacts with cell molecular oxygen to produce reactive oxygen species damaging lipids, proteins and DNA, and ultimately results in cancer cell apoptosis or necrosis ${ }^{62}$. It was found that AgNPs and AuNPs easily penetrate and accumulate in the cytosol and nucleus, which leads to inflammatory and apoptotic process activation, which in turn causes DNA damage. In addition, expression of messenger RNA plasmon(mRNA), caspase-3 protein and caspase-7, TNF- $\alpha$ and nuclear factor kappa-light-chainenhancer of activated $\mathrm{B}$ cells $(\mathrm{NF}-\mathrm{kB})$ provides induction of the internal and external apoptosis mechanism and inflammatory pathways in cells treated with AgNPs and AuNPs ${ }^{63}$. The use of AgNPs and AuNPs optoelectronic properties has given a new impetus to theranostics development in oncology, combining diagnostics and therapy. Multifunctional catalytic properties and SPR NPs of noble metals provide them with an advantage in biology and immunology. M. Sengupta et al. ${ }^{64}$ emphasize in their work that AgNPs and AuNPs do exert antitumor activity by shifting tumor-associated macrophages (TAMs) from the M2 phenotype to the M1 phenotype, as evidenced by a decrease in TNF $\alpha$ and Interleukin-10(IL-10) and a concomitant increase level of immunoregulatory cytokine IL-12. In addition, the study demonstrates TNF- $\alpha$ expression leveling after treatment with NPs, due to TAMs hypoxia reduction, which reduces the tumor volume during treatment with NPs. It was obvious that these NPs were not only immunoreactive, but also sent sensitive to oxidizing agent signals to the tumor microenvironment, inducing oxidative stress ${ }^{65}$. Microbial synthesis of biocompatible AgNPs, AuNPs and their alloys (Ag/AuNPs) for hepatoprotective activity against liver cancer caused by diethylnitrosamine also deserves attention ${ }^{66}$. The most common population in the reactive tumor stroma is fibroblasts (cancer-associated fibroblasts - CAF), since they can make up $80 \%$ of the total tumor mass, in particular, pancreas tumor ${ }^{67}$. The continuous and reciprocal information exchange between CAFs and cancer cells supports pre-metastatic niche creation, to which CAFs contribute mainly by isolating many growth factors, releasing tumor stimulating exosomes, inducing epithelialmesenchymal transitions and neoangiogenesis, and by remodeling the components of the extracellular matrix $^{68,69}$. Moreover, CAFs may contribute to the development of a multidrug-resistant tumor phenotype $^{70}$. Hybrid nanomaterial presence in the microenvironment of the tumor, in which Au is the nucleus and $\mathrm{Ag}$ is the membrane, is accompanied by a tumor cell-stimulating response weakening associated with CAFs. Intracellular presence of gold NPs Au@Ag nucleus can provide a significant advantage in cancer therapy. Thus, according to the literature, gold nuclei NPs Au@Ag have a strong radiosensitizing effect in cancer cells, which significantly increases radiotherapy effectiveness by reducing the proliferating cancer cells number in fibroblast-rich tumor microdomains ${ }^{71}$.

First described by Matsumura and Maeda in 1986, the effect of increased permeability and retention (EPR) interprets specific NPs accumulation at the tumor site ${ }^{72,73}$. The authors explained that NPs selectively accumulate in solid tumor masses as a result of certain tumor properties. Solid tumors contain leaking blood vessels with intercellular gaps of $100 \mathrm{~nm}$ to $780 \mathrm{~nm}^{74}$, compared with pore diameters of up to $20 \mathrm{~nm}$ in normal capillaries ${ }^{75-77}$. NPs with diameters up to $100 \mathrm{~nm}$ pass through the reticuloendothelial system to accumulate in the tumor $\operatorname{are}^{75,78}$. However, these endothelial fenestrations` sizes are known to vary depending on the tumor type and microenvironment ${ }^{79}$. After assembly within the tumor interstitium, NPs persist due to locally ineffective lymphatic drainage. Tumors with poor vascularization, such as pancreatic or prostate cancer, cannot accumulate $\mathrm{NPs}^{80}$. In recent years, photothermal therapy has attracted increasing attention as a fifth-line tumor treatment after surgery, chemotherapy, radiation therapy, and biological therapy ${ }^{81-83}$. This treatment involves 
nanomaterial selective accumulation with a near-infrared (NIR) photothermal focus conversion at the tumor site, which then absorbs NIR light and efficiently converts it into heat to kill the tumor cells ${ }^{84}$,

${ }^{85}$. The most important photothermal therapy advantage is that it can theoretically provide effective treatment for all solid tumors, including those that are not amenable to radiation therapy and chemotherapy, along with drug-resistant tumors ${ }^{86-88}$.

$\mathrm{Au}-\mathrm{Ag}$ bimetallic material has optical properties similar to those of AuNPs. This indicates that it can be used to detect and treat cancer due to an increase in temperature after irradiation resulting from the effect of localized surface plasmon resonance (LSPR) ${ }^{89,90}$. Thus, AuNPs and AgNPs are promising candidates for combined cancer and related infections treatment. However, the AuNPs and AgNPs potential for using antimicrobials has not yet been well studied ${ }^{91}$. It should be noted that considerable attention is paid specifically to NPs that absorb radiation in the near infrared region ${ }^{57,58}$. Among them NPsAu are most commonly used in theranostics ${ }^{59,61}$. The combination of good AuNPs biocompatibility and the ability of some of them to absorb radiation in the near-infrared NIR region (0.75-1.4 microns, the so-called biological window) make them optimal for medical use. This type of cancer treatment is based on thermal cell destruction by a sharp temperature increase after NPs irradiation in the immediate vicinity of the tumor with near infrared light. The main advantages of this therapeutic method are: minimal invasiveness for the patient and excellent penetration into the tissue (up to $10 \mathrm{~cm}$ ) ${ }^{62,92}$. Photothermal therapy research is currently focused on development of nanomaterials with high photothermal conversion efficiency. Precious metal nanomaterials, such as AuNPs with surface plasmon resonance, are considered ideal photothermal conversion materials, which leads to enhanced optical absorption $^{93}$. Compared to several conventional spherical smooth nanostructures, branched AuNPs appeared as new Au nanostructures with many advantages, including a large specific surface area, which leads to even greater photothermal transduction efficiency ${ }^{94}$. Thus, branched AuNPs and AgNPs coated with polydopamine exhibit excellent structural stability and biocompatibility. More importantly, these NPs can effectively inhibit HeLa cells growth after laser irradiation ${ }^{95}$.

Effectiveness of NPs as radiosensitizers, dose enhancers, and contrast agents is becoming increasingly recognized ${ }^{96}$. NPs are included in radiotherapy and accumulate inside the tumor. It was found that NPsAu aggregate due to interaction with glutathione in the cytosol. Compared to normal tissue more AuNPs accumulate in cancer cells, since the level of glutathione in them is much higher than in normal cells. This increases radiation therapy effectiveness, which is significantly reduced in tumors because of multidrug resistance and anti-apoptotic survivin protein action ${ }^{97}$. During treatment, when a photon beam irradiates a tumor, NPs inside the tumor interact with photons to emit more secondary electrons ${ }^{98}$. These electrons contribute to further damage to cancer cells near AuNPs by increasing the dose absorbed by the tumor ${ }^{99}$. Au has a high atomic weight $(\mathrm{Z}=79)$ and, as such, AuNPs absorb more photons than soft tissues ${ }^{100}$. It is well known that the photoelectric effect dominates in a high atomic number environment irradiated by low-energy photons in the kilovolt range ${ }^{101}$. It is the characteristic high atomic weight of NPs gold that makes them effective visualizing contrast agents ${ }^{102}$.

\section{A systematic genotoxicity and antigenotoxicity review of biologically synthesized metal nanomaterials}

Nanotoxicity as a new branch of toxicity can be characterized as a study of nanomaterial adverse effects on living organisms and ecosystems ${ }^{103}$. Genotoxicity is a problem associated with using biogenic MNPs, since they play an important role in initiation and progression of possible adverse processes for the body. Genotoxicity can be defined as point mutations, damage to genetic information within the cell, such as DNA strand breaks, chromosomal fragmentation and changes in gene expression profiles ${ }^{104}$. Genotoxins can damage the DNA sequence and chromosome structure by adding, deletion, duplication and ring formation ${ }^{105}$. Genotoxins are divided according to their action into three groups: carcinogens, mutagens and teratogens ${ }^{106}$. 
Regulators in most countries rely on specific guidelines for testing potential drugs for genotoxicity as part of their safety assessment ${ }^{107}$. Based on new nanoproducts rapid development, MNPs genotoxicity data are needed to transfer these nanoproducts from research laboratories into clinical practice. Nanomaterial physicochemical and biological properties, their small size, greatly increase their reactivity and interaction with biological tissues. In addition, NPs are able to cross biological barriers and exert their beneficial or toxic effects on the human body ${ }^{108}$. Nanogenotoxicity can be either primary or secondary. Primary nano-induced genotoxicity may be caused by NPs' direct or indirect effects on genetic material. During direct primary genotoxicity NPs interact immediately with chromosomes in the course of interphase and can bind to DNA molecules and prevent DNA replication or transcription. In addition, NPs can interact with chromosomes during mitosis, causing a chromosomal rupture (clastogenic effect) or their loss (aneugenic effect) due to mechanical or chemical actions. With indirect primary genotoxicity NPs-mediated increase in toxic ions generation and release can block proteins necessary for DNA replication and transcription ${ }^{109,}{ }^{110}$. A 2018 meta-analysis showed that biogenic NPs cytotoxicity in cancer cell lines was 9 times higher than in normal cell lines, indicating a much greater biogenic MNPs cytotoxicity in cancer cell lines $(\mathrm{OR}=9.004, \mathrm{p} 0.001)^{111}$. NPs genotoxicity can be affected by their properties, including composition, size, shape, surface characteristics, physicochemical parameters $(\mathrm{pH}$, temperature), dose, solubility, exposure time, cell type used and treatment regimen ${ }^{109,112}$. NPs composition is the main potential genotoxicity driver. Increased reactivity in contact with biological objects can induce higher ROS production. Moreover, the surface composition is a critical parameter determining the types and degree of NPs interaction with the biological system, metal ions dissolution rate and NPs biodistribution ${ }^{113}, 109$.

Numerous works of the last decade reported on MNPs green synthesis methods which use biological ways as an environmentally friendly approach. However, laboratory studies of biologically synthesized MNPs genotoxicity are still small in number and demonstrate significant heterogeneity ${ }^{114}$. Thus, MNPs are increasingly used in the biomedical field. In cancer biology MNPs are being developed for both diagnosis and therapy. NPs of metals and metal oxides can be synthesized, if necessary, and further modified by various chemical functional groups. Their functionalization helps conjugate them with biological molecules (such as antibodies, nucleic acids and peptides), ligands and antitumor drugs.

\section{References}

1. Siegel R., Naishadham D., and Jemal A. (2012) Cancer statistics. CA Cancer J Clin., 62 (1) 10-29.

2. Bray F., Jemal A., Grey N., Ferlay J., and Forman D. (2012) Global cancer transitions according to the Human Development Index (2008-2030): a population-based study. Lancet Oncol., 13 (8) 790-801.

3. Li J., Chen L., Yan L., Gu Z., Chen Z., Zhang A., and Zhao F. (2019) A Novel Drug Design Strategy: An Inspiration from Encaging Tumor by Metallofullerenol Gd@C82(OH)22. Mol Basel Switz., 24 (13) 2387.

4. Zhang Y., Li N., Suh H., and Irvine D. J. (2018) Nanoparticle anchoring targets immune agonists to tumors enabling anti-cancer immunity without systemic toxicity. Nat Commun., 9 (1) 6.

5. Xu J., Han W., Jia T., Dong S., Bi H., Yang D., He F., Dai Y., Gai S., and Yang P. (2018) Bioresponsive upconversion nanostructure for combinatorial bioimaging and chemo-photothermal synergistic therapy. Chem Eng J., 342 (342) 446-457.

6. Zhang Y., Yang D., Chen H., Lim W. Q., Phua F. S. Z., An G., Yang P., and Zhao Y. (2018) Reductionsensitive fluorescence enhanced polymeric prodrug nanoparticles for combinational photothermalchemotherapy. Biomaterials, 163 14-24.

7. Zottel A., Videtič Paska A., and Jovčevska I. (2019) Nanotechnology Meets Oncology: Nanomaterials in Brain Cancer Research, Diagnosis and Therapy. Mater Basel Switz., 12 (10) 1588.

8. Buzea C., Pacheco I. I., and Robbie K. (2007) Nanomaterials and nanoparticles: sources and toxicity. Biointerphases, 2 (4) MR17-71.

9. Saravanan M., Asmalash T., Gebrekidan A., Gebreegziabiher D., Araya T., Hilekiros H., Barabadi H., and Ramanathan K. (2018) Nano-Medicine as a Newly Emerging Approach to Combat Human Immunodeficiency Virus (HIV). Pharm Nanotechnol., 6 (1) 17-27. 
10. Barabadi H., Mahjoub M.A., Tajani B., Ahmadi A., Junejo Y., and Saravanan M. (2019) Emerging Theranostic Biogenic Silver Nanomaterials for Breast Cancer: A Systematic Review. J Clust Sci., 30 (2) 259279.

11. Ovais M., Khalil A. T., Raza A., Islam N. U., Ayaz M., Saravanan M., Ali M., Ahmad I., Shahid M., and Shinwari Z. K. (2018) Multifunctional theranostic applications of biocompatible green-synthesized colloidal nanoparticles. Appl Microbiol Biotechnol., 102 (10) 4393-4408.

12. Freitas R. A. (2005) What is nanomedicine? Nanomedicine Nanotechnol Biol Med., 1 (1) 2-9.

13. Löhr M., van der Wijngaart W., Fagerberg B. (2017) Nanoparticles for cancer therapy. Lakartidningen, 3 (2017) 114.

14. Barabadi H., Najafi M., Samadian H., Azarnezhad A., Vahidi H., Mahjoub M. A., Koohiyan M., and Ahmadi A. (2019) A Systematic Review of the Genotoxicity and Antigenotoxicity of Biologically Synthesized Metallic Nanomaterials: Are Green Nanoparticles Safe Enough for Clinical Marketing? Med Kaunas Lith., 55 (8) 439-444.

15. Wang Y., and Xia Y. (2004) Bottom-Up and Top-Down Approaches to the Synthesis of Monodispersed Spherical Colloids of Low Melting-Point Metals. Nano Lett., 4 (10) 2047-2050.

16. Ghasemi A., Rabiee N., Ahmadi S., Hashemzadeh S., Lolasi F., Bozorgomid M., Kalbasi A., Nasseri B., Shiralizadeh Dezfuli A., Aref A. R., Karimi M., and Hamblin M. R. (2018) Optical assays based on colloidal inorganic nanoparticles. The Analyst, 143 (14) 3249-3283

17. Li C., Li D., Wan G., Xu J., and Hou W. (2011) Facile synthesis of concentrated gold nanoparticles with low size-distribution in water: temperature and pH controls. Nanoscale Res Lett., 6 (1) 440.

18. Sivaraman S. K., Kumar S., and Santhanam V. (2011) Monodisperse sub-10 nm gold nanoparticles by reversing the order of addition in Turkevich method--the role of chloroauric acid. J Colloid Interface Sci., 361 (2) 543-547.

19. Kimling J., Maier M., Okenve B., Kotaidis V., Ballot H., and Plech A. (2006) Turkevich method for gold nanoparticle synthesis revisited. J Phys Chem B., 110 (32) 15700-15707.

20. Jana N. R., Gearheart L., and Murphy C. J. (2001) Seed-Mediated Growth Approach for ShapeControlled Synthesis of Spheroidal and Rod-like Gold Nanoparticles Using a Surfactant Template. Adv Mater., 13 (18) 1389-1393.

21. Fleming D. A., and Williams M. E. (2004) Size-controlled synthesis of gold nanoparticles via hightemperature reduction. Langmuir ACS J Surf Colloids., 20 (8) 3021-3023.

22. Ji X., Song X., Li J., Bai Y., Yang W., and Peng X. (2007) Size control of gold nanocrystals in citrate reduction: the third role of citrate. J Am Chem Soc., 129 (45) 13939-13948.

23. Gutiérrez-Wing C., Esparza R., Vargas-Hernández C., Fernández García M. E., and José-Yacamán M. (2012) Microwave-assisted synthesis of gold nanoparticles self-assembled into self-supported superstructures. Nanoscale, 4 (7) 2281-2287.

24. Yang S., Wang Y., Wang Q., Zhang R., and Ding B. (2007) UV irradiation induced formation of Au nanoparticles at room temperature: The case of $\mathrm{pH}$ values. Colloids Surf-Physicochem Eng Asp - COLLOID Surf A., 301 (1) 174-183.

25. Barabadi H., Honary S., Ebrahimi P., Alizadeh A., Naghibi F., and Saravanan M. (2019) Optimization of myco-synthesized silver nanoparticles by response surface methodology employing Box-Behnken design. Inorg Nano-Met Chem., 49 (2) 33-43.

26. Barabadi H. (2017) Nanobiotechnology: A promising scope of gold biotechnology. Cell Mol Biol Noisy-Gd Fr., 63 (12) 3-4.

27. Barabadi H., Kobarfard F., and Vahidi H. (2018) Biosynthesis and Characterization of Biogenic Tellurium Nanoparticles by Using Penicillium chrysogenum PTCC 5031: A Novel Approach in Gold Biotechnology. Iran J Pharm Res IJPR., 17 (Suppl2) 87-97.

28. Singh M., Harris-Birtill D. C. C., Markar S. R., Hanna G. B., and Elson D. S. (2015) Application of gold nanoparticles for gastrointestinal cancer theranostics: A systematic review. Nanomedicine Nanotechnol Biol Med., 11 (8) 2083-2098.

29. Rivas L., Sanchez-Cortes S., García-Ramos J. V., and Morcillo G. (2000) Mixed Silver/Gold Colloids: A Study of Their Formation, Morphology, and Surface-Enhanced Raman Activity. Langmuir, 16(25) 97229728.

30. Lu L., Wang H., Zhou Y., Xi S., Zhang H., Hu J. and Zhao B. (2002) Seed-mediated growth of large, monodisperse core-shell gold-silver nanoparticles with Ag-like optical properties. Chem Commun Camb Engl., 2002 (2) 144-145.

31. Doria G., Larguinho M., Dias J. T., Pereira E., Franco R., and Baptista P. V. (2010) Gold-silver-alloy nanoprobes for one-pot multiplex DNA detection. Nanotechnology, 21 (25) 255101. 
32. Sotiriou G. A., Etterlin G. D., Spyrogianni A., Krumeich F., Leroux J-C., and Pratsinis S. E. (2014) Plasmonic biocompatible silver-gold alloyed nanoparticles. Chem Commun Camb Engl., 50 (88) 1355913562.

33. Bhatia S. (2016) Nanoparticles Types, Classification, Characterization, Fabrication Methods and Drug Delivery Applications. Natural Polymer Drug Delivery Systems, Springer, Berlin.

34. Johnston R. L., and Wilcoxon J. P. (2012) Metal Nanoparticles and Nanoalloys, Vol 3, 1st Edition, Elsevier, Amsterdam.

35. De Jong W. H., and Borm P. J. A. (2008) Drug delivery and nanoparticles:applications and hazards. Int J Nanomedicine., 3 (2) 133-149.

36. Gaumet M., Vargas A., Gurny R., and Delie F. (2008) Nanoparticles for drug delivery: the need for precision in reporting particle size parameters. Eur $J$ Pharm Biopharm Off J Arbeitsgemeinschaft Pharm Verfahrenstechnik EV., 69 (1) 1-9.

37. Kumar C. S. S. R., ed. (2013) Geometrically Tunable Optical Properties of Metal Nanoparticles. UVVIS and Photoluminescence Spectroscopy for Nanomaterials Characterization, Springer, Berlin.

38. Joshi M., Bhattacharyya A., and Ali S. W. (2008) Characterization techniques for nanotechnology applications in textiles. IJFTR., 33 (3) 304-317.

39. Zheng T., Bott S., and Huo Q. (2016) Techniques for Accurate Sizing of Gold Nanoparticles Using Dynamic Light Scattering with Particular Application to Chemical and Biological Sensing Based on Aggregate Formation. ACS Appl Mater Interfaces., 8 (33) 21585-21594.

40. Chu B., and Liu T. (2000) Characterization of Nanoparticles by Scattering Techniques. J Nanoparticle Res., 2 (1) 29-41.

41. Lin P-C., Lin S., Wang P. C., and Sridhar R. (2014) Techniques for physicochemical characterization of nanomaterials. Biotechnol Adv., 32 (4) 711-726.

42. Bootz A., Vogel V., Schubert D., and Kreuter J. (2004) Comparison of scanning electron microscopy, dynamic light scattering and analytical ultracentrifugation for the sizing of poly (butyl cyanoacrylate) nanoparticles. Eur J Pharm Biopharm Off J Arbeitsgemeinschaft Pharm Verfahrenstechnik EV., 57 (2) 369375.

43. Zhang X-F., Liu Z-G., Shen W., and Gurunathan S. (2016) Silver Nanoparticles: Synthesis, Characterization, Properties, Applications, and Therapeutic Approaches. Int J Mol Sci., 17 (9) 1468.

44. Caputo F., De Nicola M., and Ghibelli L. (2014) Pharmacological potential of bioactive engineered nanomaterials. Biochem Pharmacol., 92 (1) 112-130.

45. Chugh H., Sood D., Chandra I., Tomar V., Dhawan G., and Chandra R. (2018) Role of gold and silver nanoparticles in cancer nano-medicine. Artif Cells Nanomedicine Biotechnol., 46 (sup1) 1210-1220.

46. Greulich C., Diendorf J., Simon T., Eggeler G., Epple M., and Köller M. (2011) Uptake and intracellular distribution of silver nanoparticles in human mesenchymal stem cells. Acta Biomater., 7 (1) 347-354.

47. Jeyaraj M., Sathishkumar G., Sivanandhan G., MubarakAli D., Rajesh M., Arun R., Kapildev G., Manickavasagam M., Thajuddin N., Premkumar K., and Ganapathi A. (2013) Biogenic silver nanoparticles for cancer treatment: an experimental report. Colloids Surf B Biointerfaces., 106 86-92.

48. Kovács D., Igaz N., Marton A., Rónavári A., Bélteky P., Bodai L., Spengler G., Tiszlavicz L., Rázga Z., Hegyi P., Vizler C., Boros I. M., Kónya Z., and Kiricsi M. (2020) Core-shell nanoparticles suppress metastasis and modify the tumour-supportive activity of cancer-associated fibroblasts. J Nanobiotechnology., 18 (1) 18.

49. Liu F., Mahmood M., Xu Y., Watanabe F., Biris A. S., Hansen D. K., Inselman A., Casciano D., Patterson T. A., Paule M. G., Slikker W. Jr., and Wang C. (2015) Effects of silver nanoparticles on human and rat embryonic neural stem cells. Front Neurosci., 9 (2015) 115.

50. Zhang X-F., Shen W., and Gurunathan S. (2016) Silver Nanoparticle-Mediated Cellular Responses in Various Cell Lines: An in Vitro Model. Int J Mol Sci., 17 (10) 1603.

51. Park E-J., Yi J., Kim Y., Choi K., and Park K. (2010) Silver nanoparticles induce cytotoxicity by a Trojanhorse type mechanism. Toxicol Vitro Int J Publ Assoc BIBRA., 24 (3) 872-878.

52. Riaz Ahmed K. B., Nagy A. M., Brown R. P., Zhang Q., Malghan S. G., and Goering P. L. (2017) Silver nanoparticles: Significance of physicochemical properties and assay interference on the interpretation of in vitro cytotoxicity studies. Toxicol Vitro Int J Publ Assoc BIBRA., 38 179-192.

53. Carlson C., Hussain S. M., Schrand A. M., Braydich-Stolle L. K., Hess K. L., Jones R. L., and Schlager J. J. (2008) Unique cellular interaction of silver nanoparticles: size-dependent generation of reactive oxygen species. J Phys Chem B., 112 (43) 13608-13619.

54. Gurunathan S., Lee K-J., Kalishwaralal K., Sheikpranbabu S., Vaidyanathan R., and Eom S. H. (2009) Antiangiogenic properties of silver nanoparticles. Biomaterials, 30 (31) 6341-6350. 
55. Asharani P. V., Hande M. P., and Valiyaveettil S. (2009) Anti-proliferative activity of silver nanoparticles. BMC Cell Biol., 10 (2009) 65.

56. Mukherjee P., Bhattacharya R., Wang P., Wang L., Basu S., Nagy J. A., Atala A., Mukhopadhyay D., and Soker S. (2005) Antiangiogenic properties of gold nanoparticles. Clin Cancer Res Off J Am Assoc Cancer Res., 11 (9) 3530-3534.

57. Zhang Y., Ren K., Zhang X., Chao Z., Yang Y., Ye D., Dai Z., Liu Y., and Ju H. (2018) Photo-tearable tape close-wrapped upconversion nanocapsules for near-infrared modulated efficient siRNA delivery and therapy. Biomaterials, 163 55-66.

58. Liu H., Li W., Cao Y., Guo Y., and Kang Y. (2018) Theranostic nanoplatform based on polypyrrole nanoparticles for photoacoustic imaging and photothermal therapy. J Nanoparticle Res., 20 (3) 57.

59. Norouzi H., Khoshgard K., and Akbarzadeh F. (2018) In vitro outlook of gold nanoparticles in photothermal therapy: a literature review. Lasers Med Sci., 33 (4) 917-926.

60. Zhou G., Xiao H., Li X., Huang Y., Song W., Song L., Chen M., Cheng D., and Shuai X. (2017) Gold nanocage decorated $\mathrm{pH}$-sensitive micelle for highly effective photothermo-chemotherapy and photoacoustic imaging. Acta Biomater., 64 223-236.

61. Chauhan G., Chopra V., Tyagi A., Rath G., Sharma R. K., and Goyal A. K. (2017) "Gold nanoparticles composite-folic acid conjugated graphene oxide nanohybrids" for targeted chemo-thermal cancer ablation: In vitro screening and in vivo studies. Eur J Pharm Sci Off J Eur Fed Pharm Sci., 96 351-361.

62. Hirschberg H., and Madsen S. J. (2017) Cell Mediated Photothermal Therapy of Brain Tumors. $J$ Neuroimmune Pharmacol Off J Soc NeuroImmune Pharmacol., 12 (1) 99-106.

63. Sivakumar A.S., Krishnaraj C., Sheet S., Rampa D. R., Kang D. R., Belal S. A., Kumar A., Hwang I. H., Yun S. I., Lee Y. S., and Shim K. S. (2017) Interaction of silver and gold nanoparticles in mammalian cancer: as real topical bullet for wound healing- A comparative study. In Vitro Cell Dev Biol Anim., 53 (7) 632-645.

64. Sengupta M., Pal R., Nath A., Chakraborty B., Singh L. M., Das B., and Ghosh S. K. (2018) Anticancer efficacy of noble metal nanoparticles relies on reprogramming tumor-associated macrophages through redox pathways and pro-inflammatory cytokine cascades. Cell Mol Immunol., 15 (12) 1088-1090.

65. Pal R., Chakraborty B., Nath A., Singh L. M., Ali M., Rahman D. S., Ghosh S. K., Basu A., Bhattacharya S., Baral R., and Sengupta M. (2016) Noble metal nanoparticle-induced oxidative stress modulates tumor associated macrophages (TAMs) from an M2 to M1 phenotype: An in vitro approach. Int Immunopharmacol., 38 332-341.

66. Shanmugasundaram T., Radhakrishnan M., Gopikrishnan V., Kadirvelu K., and Balagurunathan R. (2017) Biocompatible silver, gold and silver/gold alloy nanoparticles for enhanced cancer therapy: in vitro and in vivo perspectives. Nanoscale, 9 (43) 16773-16790.

67. Fiori M. E., Di Franco S., Villanova L., Bianca P., Stassi G., and De Maria R. (2019) Cancer-associated fibroblasts as abettors of tumor progression at the crossroads of EMT and therapy resistance. Mol Cancer., 18 (1) 70.

68. Li W., Zhang X., Wang J., Li M., Cao C., Tan J., Ma D., and Gao Q. (2017) TGF $\beta 1$ in fibroblasts-derived exosomes promotes epithelial-mesenchymal transition of ovarian cancer cells. Oncotarget, 8 (56) 9603596047.

69. Richards K. E., Zeleniak A. E., Fishel M. L., Wu J., Littlepage L. E., and Hill R. (2017) Cancer-associated fibroblast exosomes regulate survival and proliferation of pancreatic cancer cells. Oncogene, 36 (13) 17701778.

70. Ireland L.V., and Mielgo A. (2018) Macrophages and Fibroblasts, Key Players in Cancer Chemoresistance. Front Cell Dev Biol., 6 (2108) 131.

71. Peukert D., Kempson I., Douglass M., and Bezak E. (2018) Metallic nanoparticle radiosensitisation of ion radiotherapy: A review. Phys Medica PM Int J Devoted Appl Phys Med Biol Off J Ital Assoc Biomed Phys AIFB., 47 (2018) 121-128.

72. Maeda H. (2010) Tumor-selective delivery of macromolecular drugs via the EPR effect: background and future prospects. Bioconjug Chem., 21 (5) 797-802.

73. Matsumura Y., and Maeda H. (1986) A new concept for macromolecular therapeutics in cancer chemotherapy: mechanism of tumoritropic accumulation of proteins and the antitumor agent smancs. Cancer Res., 46 (12 Pt 1) 6387-6392.

74. Kommareddy S., Tiwari S. B., and Amiji M. M. (2005) Long-circulating polymeric nanovectors for tumor-selective gene delivery. Technol Cancer Res Treat., 4 (6) 615-625.

75. Sykes P. D., Neoptolemos J. P., Costello E., and Halloran C. M. (2012) Nanotechnology advances in upper gastrointestinal, liver and pancreatic cancer. Expert Rev Gastroenterol Hepatol., 6 (3) 343-356. 
76. Yuan F., Dellian M., Fukumura D., Leunig M., Berk D. A., Torchilin V. P., and Jain R. K. (1995) Vascular permeability in a human tumor xenograft: molecular size dependence and cutoff size. Cancer Res., 55 (17) 3752-3756.

77. Firth J. A. (2002) Endothelial barriers: from hypothetical pores to membrane proteins. J Anat., 200 (6) 541-548.

78. Perrault S. D., Walkey C., Jennings T., Fischer H. C., and Chan W. C. W. (2009) Mediating tumor targeting efficiency of nanoparticles through design. Nano Lett., 9 (5) 1909-1915.

79. Hobbs S. K., Monsky W. L., Yuan F., Roberts W. G., Griffith L., Torchilin V. P., and Jain R. K. (1998) Regulation of transport pathways in tumor vessels: role of tumor type and microenvironment. Proc Natl Acad Sci U S A., 95 (8) 4607-4612.

80. Hu Z., Huo F., Zhang Y., Chen C., Tu K., Wang H. J. and Wang L-Q. (2011) "Smart” Nanocarriers: A New Paradigm for Tumor Targeting Drug Delivery Systems. Drug Deliv Lett., 1 (1) 67.

81. Zhang C-L., Lv K-P., Cong H-P., and Yu S-H. (2020) Controlled Assemblies of Gold Nanorods in PVA Nanofiber Matrix as Flexible Free-Standing SERS Substrates by Electrospinning. Small Weinh Bergstr Ger., 16 (2) e1904785.

82. Tian G., Zhang X., Gu Z., and Zhao Y. (2015) Recent Advances in Upconversion Nanoparticles-Based Multifunctional Nanocomposites for Combined Cancer Therapy. Adv Mater Deerfield Beach Fla., 27 (47) $7692-7712$.

83. Zhao X., Qi T., Kong C., Hao M., Wang Y., Li J., Liu B., Gao Y., and Jiang J. (2018) Photothermal exposure of polydopamine-coated branched $\mathrm{Au}-\mathrm{Ag}$ nanoparticles induces cell cycle arrest, apoptosis, and autophagy in human bladder cancer cells. Int J Nanomedicine., 13 (2018) 6413-6428.

84. Liu J., Zheng X., Yan L., Zhou L., Tian G., Yin W., Wang L., Liu Y., Hu Z., Gu Z., Chen C., and Zhao Y. (2015) Bismuth sulfide nanorods as a precision nanomedicine for in vivo multimodal imaging-guided photothermal therapy of tumor. ACS Nano., 9 (1) 696-707.

85. Norman R. S., Stone J. W., Gole A., Murphy C .J., and Sabo-Attwood T. L. (2008) Targeted photothermal lysis of the pathogenic bacteria, Pseudomonas aeruginosa, with gold nanorods. Nano Lett., 8 (1) 302-306.

86. Shi J., Wang L., Zhang J., Ma R., Gao J., Liu Y., Zhang C., and Zhang Z. (2014) A tumor-targeting nearinfrared laser-triggered drug delivery system based on GO@Ag nanoparticles for chemo-photothermal therapy and X-ray imaging. Biomaterials, 35 (22) 5847-5861.

87. Guo F., Yu M., Wang J., Tan F., and Li N. (2015) Smart IR780 Theranostic Nanocarrier for TumorSpecific Therapy: Hyperthermia-Mediated Bubble-Generating and Folate-Targeted Liposomes. ACS Appl Mater Interfaces., 7 (37) 20556-20567.

88. Sheng W., He S., Seare W.J., and Almutairi A. (2017) Review of the progress toward achieving heat confinement-the holy grail of photothermal therapy. J Biomed Opt., 22 (8) 80901.

89. Nima Z. A., Mahmood M., Xu Y., Mustafa T., Watanabe F., Nedosekin D. A., Juratli M. A., Fahmi T., Galanzha E. I., Nolan J. P., Basnakian A. G., Zharov V. P., and Biris A. S. (2014) Circulating tumor cell identification by functionalized silver-gold nanorods with multicolor, super-enhanced SERS and photothermal resonances. Sci Rep., 4 (2014) 4752.

90. Nikoobakht B., Wang J., and El-Sayed M. (2002) Surface-enhanced Raman scattering of molecules adsorbed on gold nanorods: Off-surface plasmon resonance condition. Chem Phys Lett., 366 (1) 17-23.

91. Zhang J., Wang M., and Webster T. J. (2018) Silver-coated gold nanorods as a promising antimicrobial agent in the treatment of cancer-related infections. Int J Nanomedicine., 13 (2018) 6575-6583.

92. Rajkumar S., and Prabaharan M. (2017) Theranostics Based on Iron Oxide and Gold Nanoparticles for Imaging- Guided Photothermal and Photodynamic Therapy of Cancer. Curr Top Med Chem., 17 (16) 18581871.

93. Ungureanu C., Kroes R., Petersen W, Groothuis T. A. M., Ungureanu F., Janssen H., van Leeuwen F. W. B, Kooyman R. P. H., Manohar S., and van Leeuwen T. G. (2011) Light interactions with gold nanorods and cells: implications for photothermal nanotherapeutics. Nano Lett., 11 (5) 1887-1894.

94. Liang S., Li C., Zhang C., Chen Y., Xu L., Bao C., Wang X., Liu G., Zhang F. and Cui D.. (2015) CD44v6 Monoclonal Antibody-Conjugated Gold Nanostars for Targeted Photoacoustic Imaging and Plasmonic Photothermal Therapy of Gastric Cancer Stem-like Cells. Theranostics, 5 (9) 970-984.

95. Li J., Wang W., Zhao L., Rong L., Lan S., Sun H., Zhang H., and Yang B. (2015) Hydroquinone-assisted synthesis of branched au-ag nanoparticles with polydopamine coating as highly efficient photothermal agents. ACS Appl Mater Interfaces., 7 (21) 11613-11623.

96. Chow J. C. L. (2017) Dose enhancement effect in radiotherapy: adding gold nanoparticles to tumor in cancer treatment. In: Ficai A., and Grumezescu A. M., eds. Nanostructures for Cancer Therapy. Elsevier, Amsterdam. 
97. Stobiecka M., Ratajczak K., and Jakiela S. (2019) Toward early cancer detection: Focus on biosensing systems and biosensors for an anti-apoptotic protein survivin and survivin mRNA. Biosens Bioelectron., 137 (2019) 58-71.

98. Chow J. C. L. (2016) Photon and electron interactions with gold nanoparticles: A Monte Carlo study on gold nanoparticle-enhanced radiotherapy. In: Grumezescu A. M., ed. Nanobiomaterials in Medical Imaging. William Andrew Publishing, Norwich.

99. Cai Q. Y., Kim S. H., Choi K. S., Kim S. Y., Byun S. J., Kim K. W., Park S. H., Juhng S. K., and Yoon K. H. (2007) Colloidal gold nanoparticles as a blood-pool contrast agent for X-ray computed tomography in mice. Invest Radiol., 42 (12) 797-806.

100. Rogers D. W. O. (2002) Monte Carlo Techniques in Radiotherapy. Phys Can., 58 (2) 63-70.

101. Cormode D. P., Naha P. C., and Fayad Z. A. (2014) Nanoparticle contrast agents for computed tomography: a focus on micelles. Contrast Media Mol Imaging., 9 (1) 37-52.

102. Abdulle A., and Chow J. C. L. (2019) Contrast Enhancement for Portal Imaging in NanoparticleEnhanced Radiotherapy: A Monte Carlo Phantom Evaluation Using Flattening-Filter-Free Photon Beams. Nanomater Basel Switz., 9 (7) 920.

103. Oberdörster G. (2010) Safety assessment for nanotechnology and nanomedicine: concepts of nanotoxicology. J Intern Med., 267 (1) 89-105.

104. Xie H., Mason M. M., and Wise J. P. (2011) Genotoxicity of metal nanoparticles. Rev Environ Health., 26 (4) 251-268.

105. Seukep A. J., Noumedem J. A. K., Djeussi D. E., and Kuete V. (2014) 9 - Genotoxicity and Teratogenicity of African Medicinal Plants. In: Kuete V., ed. Toxicological Survey of African Medicinal Plants. Elsevier, Amsterdam.

106. Mohamed S., Sabita U., Rajendra S., and Raman D. (2017) Genotoxicity: Mechanisms, Testing Guidelines and Methods. Glob J Pharmaceu Sci. 1 (5) 1-6.

107. Rama R. P., Kaul C. L., and Jena G. B. (2002) Genotoxicity testing, a regulatory requirement for drug discovery and development: Impact of ICH guidelines. Indian J Pharmacol.,34 (2) 86.

108. Amoabediny G. H., Naderi A., Malakootikhah J., Koohi M. K., Mortazavi S. A., Naderi M. and Rashedi H. (2009) Guidelines for safe handling, use and disposal of nanoparticles. J Phys Conf Ser., 170 (2009) 012037.

109. Magdolenova Z., Collins A., Kumar A., Dhawan A., Stone V., and Dusinska M. (2014) Mechanisms of genotoxicity. A review of in vitro and in vivo studies with engineered nanoparticles. Nanotoxicology, 8 (3) 233-278.

110. Mahaye N., Thwala M., Cowan D. A., and Musee N. (2017) Genotoxicity of metal based engineered nanoparticles in aquatic organisms: A review. Mutat Res., 773 (2017) 134-160.

111. Barabadi H., Alizadeh A., Ovais M., Ahmadi A., Shinwari Z. K., and Saravanan M. (2018) Efficacy of green nanoparticles against cancerous and normal cell lines: a systematic review and meta-analysis. IET Nanobiotechnol., 12 (4) 377-391.

112. Stone V., Johnston H., and Schins R. P. F. (2009) Development of in vitro systems for nanotoxicology: methodological considerations. Crit Rev Toxicol., 39 (7) 613-626.

113. Sharifi S., Behzadi S., Laurent S., Forrest M. L., Stroeve P., and Mahmoudi M. (2012) Toxicity of nanomaterials. Chem Soc Rev., 41 (6) 2323-2343.

114. Mody V. V., Siwale R., Singh A., and Mody H. R. (2010) Introduction to metallic nanoparticles. $J$ Pharm Bioallied Sci., 2 (4) 282-289.

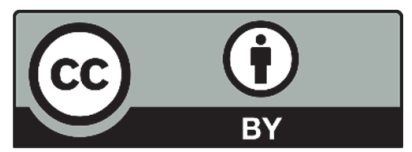

(C) 2021 by the authors; licensee Growing Science, Canada. This is an open access article distributed under the terms and conditions of the Creative Commons Attribution (CC-BY) license (http://creativecommons.org/licenses/by/4.0/). 